\title{
Towards a world-sheet description of doubled geometry in string theory
}

\author{
Ioannis Bakas $^{1}$, Dieter Lüst ${ }^{2,3}$, Erik Plauschinn ${ }^{3}$ \\ ${ }^{1}$ Department of Physics \\ School of Applied Mathematics and Physical Sciences \\ National Technical University \\ 15780 Athens, Greece \\ ${ }^{2}$ Max-Planck-Institut für Physik \\ Föhringer Ring 6 \\ 80805 München, Germany \\ ${ }^{3}$ Arnold-Sommerfeld-Center für Theoretische Physik \\ Department für Physik \\ Ludwig-Maximilians-Universität München \\ Theresienstraße 3\%, 80333 München, Germany
}

\begin{abstract}
Starting from a sigma-model for a doubled target-space geometry, we show that the number of target-space dimensions can be reduced by half through a gauging procedure. We apply this formalism to a class of backgrounds relevant for double field theory, and illustrate how choosing different gaugings leads to string-theory configurations T-dual to each other. We furthermore discuss that given a conformal doubled theory, the reduced theories are conformal as well.

As an example we consider the three-dimensional $S U(2)$ WZW model and show that the only possible reduced backgrounds are the cigar and trumpet CFTs in two dimensions, which are indeed T-dual to each other.
\end{abstract}




\section{Contents}

1 Introduction

2 Dimensional reduction by gauging isometries 5

2.1 Gauging an isometry . . . . . . . . . . . . 5

2.2 Reduction of dimensions . . . . . . . . . . . . . . 7

2.3 Generalization to multiple isometries . . . . . . . . . . . . . 10

2.4 Summary and discussion . . . . . . . . . . . . . . 13

3 Relation to Roček \& Verlinde 15

3.1 Single doubled dimension . . . . . . . . . . . . . . . 15

3.2 Conformal invariance of parent and child theories . . . . . . . . 18

3.3 Multiple doubled dimensions . . . . . . . . . . . . . . . . 20

4 Examples

$4.1 \quad S U(2)$ WZW model . . . . . . . . . . . . . . . . . . . . 23

4.2 General WZW model . . . . . . . . . . . . . . . . . . . 24

5 Summary and conclusions

A Requirement of vanishing field strength 29

\section{Introduction}

One of string theory's attractive features is its rich structure of dualities [1]. This implies that two seemingly different formulations of string theory can be physically equivalent. An example for such a duality is T-duality, where string theory compactified on a circle of radius $R$ is equivalent to a compactification on a circle of radius $\alpha^{\prime} / R$. Another example is S-duality, which relates a weakly-coupled regime to a strongly-coupled one. Furthermore, the AdS/CFT duality makes a connection between a gravity theory on AdS spaces and conformal field theories on their boundaries; and there are many more duality relations relevant for string theory.

In this paper we are interested in T-duality, for which an extensive review can be found in [2]. More concretely, when compactifying string theory on a circle of radius $R$, the theory can be quantized and the spectrum can be determined explicitly. T-duality then means that the spectrum is invariant under the map $R \rightarrow \alpha^{\prime} / R$. For curved backgrounds, it is in general not known how to perform the quantization, however, the mapping between T-dual configurations is often still possible and is provided by the Buscher rules [3 [5]. Furthermore, Roček and 
Verlinde have shown in [6] that T-duality is not only a symmetry of the spectrum, but a true symmetry of the underlying conformal field theory. Other related work in this context can be found in [7-15].

An interesting application of dualities is that novel solutions with potentially new features can be obtained from existing ones. The prime example for this idea in the case of T-duality is the three-torus with $H$-flux, which we briefly review. Applying a T-duality transformation to this background gives the twisted torus [16,17], which is a geometric space carrying a so-called geometric flux. After a second duality transformation a locally geometric configuration is obtained, which is however globally non-geometric [18]. Here, the transition functions between different coordinate patches are no longer only diffeomorphisms, but involve duality transformations [19]. This construction is called a T-fold [20], and it carries a socalled $Q$-flux [21]. It has also been argued that a third T-duality transformation can (at least formally) be performed ending in a space with $R$-flux [21]. The nongeometric spaces spaces carry non-commutative and non-associative structures and have been studied from a mathematical point of view in [22 25], later in [26, 27], and have been reconsidered in a series of papers [28 43]. Thus, as mentioned above, applying T-duality transformations to known backgrounds can lead to new string-theory configurations with novel features.

There are a number of different approaches to study non-geometric backgrounds in particular, and T-duality in general. From the word-sheet point of view, leftright asymmetric string CFT constructions, which have been already constructed several years ago [44 47], correspond to non-geometric string backgrounds. Further aspects of non-geometric string constructions have been analyzed from a worldsheet point of view in [48 53. Non-geometric flux configurations have been investigated via field redefinitions for the ten-dimensional supergravity action in [54 61]. Another strategy is to make T-duality into a manifest symmetry of an action. This can be achieved by introducing additional auxiliary, but unphysical, coordinates. Through this procedure the dimension of space-time is doubled - and the physical configuration is obtained by removing half of the coordinates. Different choices for which coordinates are removed then lead to backgrounds which are T-dual to each other.

- From the world-sheet point of view, such a construction has first appeared in [62,63], based on work in [64, and has been revisited for instance in [65] (see also [66]). In this formulation manifest Lorentz invariance is however broken, which has later been addressed in [67, 68]. In [20, 69, 70] a different doubled formalism appeared, in which a constraint to reduce the number of coordinates has to be imposed by hand.

- From a target-space perspective, an action invariant under T-duality has been developed (using also world-sheet techniques) in [71,72], but also here 
at the cost of broken Lorentz covariance. In [73 75], combining insight from string field theory with the doubled formalism, the framework of double field theory (DFT) has been developed (for reviews see [76, 77]). Double field theory is a field theory with a doubled number of target-space dimensions, which is manifestly invariant under T-duality. Though, on the other hand, it is in general not known how to incorporate massive excitations of the string into this setting.

- We also mention that from a mathematical point of view, the idea to double the coordinates in order to make T-duality a symmetry has been discussed in [78, 79].

In this paper, we follow the approach of doubling the number of target-space dimensions in order to make T-duality a manifest symmetry. We consider a worldsheet formulation, and we discuss the conformal properties of the corresponding doubled and physical theories. Our main strategy is the following:

1. We start from a background in $D$ dimensions with two directions of isometry. The background is required to have vanishing $\beta$-functionals for the metric and $B$-field, but the $\beta$-functional for the dilaton is non-zero at tree-level. It is therefore not a critical string theory.

2. Next, we gauge one of the two isometries in the world-sheet theory, and integrate-out the corresponding gauge field. As we show in detail, the resulting metric and $B$-field are that of a $(D-1)$-dimensional target-space theory. The reduced theory in $D-1$ dimensions is conformal, in particular it is a string theory.

3. Gauging a different isometry leads to a different $D$-dimensional background. However, these lower-dimensional theories are related to each other through T-duality transformations.

Thus, we provide a world-sheet approach to doubled geometry, in which the Tdual backgrounds originate from different choices in a reduction procedure. The novel aspect of our analysis is that the reduction is achieved by gauging isometries and integrating-out the corresponding gauge field, instead of imposing constraints explicitly.

We also would like to point out relations between our work and other approaches. First, our strategy is closely related to Buscher's procedure of gauging an isometry and integrating-out the gauge field $A$ [3 [5]. However, in Buscher's work a Lagrange multiplier is introduced which implements the vanishing of the field strength $F=d A$. This Lagrange multiplier becomes the dual coordinate after T-duality, and hence the dimensionality of the target space is unchanged. In our 
work, we impose the vanishing of the field strength by hand, without including a Lagrange multiplier. We therefore find that the number of space-time dimensions is reduced. Second, there is no direct connection to the canonical-transformation approach to T-duality discussed for instance in [7,11], since we obtain a reduction of the dimensionality of the target-space. Third, similar to Buscher's approach, a Killing vector field $k$ and a one-form $\mathrm{v}$ will play a prominent role in our analysis. In particular, T-duality in the reduced theories will be realized by interchanging the role of $k$ and $v$. Such a transformation can be described using the framework of generalized geometry (see [80] and [81] for the original work, and for instance to [82] for a discussion in the context of T-duality), in which the tangent and co-tangent space are treated on equal footing. Finally, we mention that a related world-sheet discussion has appeared in [67,68].

This paper is organized as follows: in section 2 we discuss how by gauging certain symmetries of a non-linear sigma-model and integrating-out the corresponding gauge field, the number of target-space dimensions is reduced. We first consider the case of a single doubled dimension, and then generalize to multiple doubled dimensions. In section 3, we apply this procedure to a particular type of backgrounds relevant for double field theory. These backgrounds have also appeared in the work of Roček and Verlinde [6], and we find that our results incorporate those of [6] nicely. In section 4 we first discuss the $S U(2)$ Wess-Zumino-Witten model as an example, and then comment on the generalization to arbitrary Lie groups. Section 5 contains our summary and conclusions.

\section{Dimensional reduction by gauging isometries}

In this section we discuss on general grounds how by gauging a symmetry of a nonlinear sigma-model and integrating-out the gauge field, the number of target-space dimensions can be reduced.

\subsection{Gauging an isometry}

Let us start by reviewing the gauging procedure for non-linear sigma-models. This has been discussed by Hull and Spence in [83, 84, and has recently been revisited and further analyzed in [13, 14].

\section{Sigma-model action and isometries}

Our starting point is the a non-linear sigma-model action for a metric $G$, antisymmetric tensor field $B$ and a dilaton $\Phi$. We employ a formulation of this action as 
a Wess-Zumino-Witten (WZW) model, where instead of $B$ itself the field strength $H=d B$ appears. The action reads

$$
\begin{aligned}
\mathcal{S}= & -\frac{1}{4 \pi \alpha^{\prime}} \int_{\partial \Sigma}\left[G_{i j} d X^{i} \wedge \star d X^{j}+\alpha^{\prime} R \Phi \star 1\right] \\
& -\frac{i}{2 \pi \alpha^{\prime}} \int_{\Sigma} \frac{1}{3 !} H_{i j k} d X^{i} \wedge d X^{j} \wedge d X^{k}
\end{aligned}
$$

where the indices $i, j, k=1, \ldots, D$ label the target-space coordinates. The Hodge star operator on the world-sheet is denoted by $\star, \Sigma$ is a three-manifold with boundary $\partial \Sigma$, and $R$ denotes the Ricci curvature scalar on the world-sheet $\partial \Sigma$.

In order to follow the procedure we have in mind, we assume the action (2.1) to be invariant under the following global variation

$$
\delta_{\epsilon} X^{i}=\epsilon k^{i}(X), \quad \epsilon=\text { const. }
$$

This requirement translates into three conditions for the target-space metric $G$, the field strength $H$ and the dilaton $\Phi$, which read as follows:

- First, the vector $k=k^{i} \partial_{i}$ has to be a Killing vector for the metric $G$. Using the coordinate-free notation $G=G_{i j} d X^{i} \wedge \star d X^{j}$ and the Lie derivative $\mathcal{L}_{k}=d \circ \iota_{k}+\iota_{k} \circ d$, this reads

$$
\mathcal{L}_{k} G=0
$$

- Second, invariance under global variations (2.2) implies for the field strength

$$
\iota_{k} H=d \mathrm{v}
$$

for $v$ a one-form on the target space. Note that $v$ in (2.4) is defined only up to a closed part. Equation (2.4) is equivalent to $\mathcal{L}_{k} B=d \mathrm{v}^{\prime}$ with $\mathrm{v}^{\prime}$ some other one-form, which then leads to

$$
\mathcal{L}_{k} H=0
$$

- The third condition for the variation of the action to vanish is

$$
\mathcal{L}_{k} \Phi=k^{m} \partial_{m} \Phi=0
$$




\section{Gauging the sigma-model}

Next, we gauge the symmetry (2.2) by allowing $\epsilon$ to have a non-trivial dependence on the world-sheet coordinates. We therefore introduce a gauge field $A$ and replace $d X^{i} \rightarrow d X^{i}+k^{i} A$ for the term involving the metric. For the Wess-Zumino term we keep $d X^{i}$ unchanged, but introduce a coupling between the one-form $v$ and the gauge field $A$. The resulting gauge-invariant action takes the following form $[83,84$

$$
\begin{aligned}
\widehat{\mathcal{S}}= & -\frac{1}{4 \pi \alpha^{\prime}} \int_{\partial \Sigma}\left[G_{i j}\left(d X^{i}+k^{i} A\right) \wedge \star\left(d X^{j}+k^{j} A\right)+2 i \vee \wedge A+\alpha^{\prime} R \Phi \star 1\right] \\
& -\frac{i}{2 \pi \alpha^{\prime}} \int_{\Sigma} \frac{1}{3 !} H_{i j k} d X^{i} \wedge d X^{j} \wedge d X^{k},
\end{aligned}
$$

where $k$ denotes again the Killing vector of the target-space isometry which has been gauged. The symmetry transformations for the fields in the action read

$$
\hat{\delta}_{\epsilon} X^{i}=\epsilon k^{i}(X), \quad \hat{\delta}_{\epsilon} A=-d \epsilon .
$$

Moreover, for gauge invariance of the gauged action (2.7) we have to require [83,84]

$$
\iota_{k} \mathrm{v}=k^{m} \mathbf{v}_{m}=0 \text {. }
$$

Note that by gauging the symmetry (2.2) we have introduced two new degrees of freedom into the theory, out of which one can be eliminated by a gauge transformation (2.8). In order to eliminate the second additional degree of freedom, and for the gauged action (2.7) to be equivalent to the ungauged one (2.1), we impose the constraint

$$
0=F=d A
$$

When studying T-duality, this constraint is usually realized using a Lagrange multiplier. However, here we chose to impose the constraint by hand.

\subsection{Reduction of dimensions}

After gauging an isometry of world-sheet action, we now want to integrate-out the corresponding gauge field. This leads to a reduction of the number of target-space dimensions.

\section{Integrating-out the gauge field}

To integrate-out the gauge field $A$, we determine its equation of motion following from (2.7). With $|k|^{2}=k^{i} G_{i j} k^{j}$ the norm of the Killing vector field, we find

$$
|k|^{2} A=-k^{i} G_{i j} d X^{j}-i \star v .
$$


If $|k|^{2}$ is non-vanishing, we can solve (2.11) for $A$ and substitute the solution back into the action. The resulting world-sheet action takes the general form

$$
\begin{aligned}
\check{\mathcal{S}}= & -\frac{1}{4 \pi \alpha^{\prime}} \int_{\partial \Sigma}\left[\check{G}_{i j} d X^{i} \wedge \star d X^{j}+\alpha^{\prime} R \Phi \star 1\right] \\
& -\frac{i}{2 \pi \alpha^{\prime}} \int_{\Sigma} \frac{1}{3 !} \check{H}_{i j k} d X^{i} \wedge d X^{j} \wedge d X^{k} .
\end{aligned}
$$

With $\mathrm{k}=k^{i} G_{i j} d x^{j}$ the one-form dual to the Killing vector $k$, we find for $\check{G}$ and $\check{H}$ the expressions

$$
\check{G}=G-\frac{1}{|k|^{2}} \mathrm{k} \wedge \star \mathrm{k}+\frac{1}{|k|^{2}} \mathrm{v} \wedge \star \mathrm{v}, \quad \check{H}=H+d\left(\frac{1}{|k|^{2}} \mathrm{k} \wedge \mathrm{v}\right) .
$$

Let us also recall that the gauge field $A$ is subject to the constraint (2.10), in particular, the solution (2.11) has to satisfy $d A=0$. Using the equations of motion for $X^{i}$, we can express this constraint as 1

$$
0=\nabla_{\left[\underline{i} \mathrm{k}_{\underline{j}]}\right.}-\frac{1}{2} v^{m} H_{m i j}, \quad \quad 0=\nabla_{\left(\bar{i} \overline{\mathrm{v}}_{\bar{j}}\right)}, \quad v^{m} \partial_{m} \Phi=0
$$

where the Levi-Civita connection appearing in $\nabla$ is computed using the original metric $G$, and $v^{i}=G^{i j} \mathbf{v}_{j}$ are the components of the vector field $v=v^{i} \partial_{i}$ dual to the one-form v. Details about the derivation of these relations can be found in appendix A.

\section{Reduced geometry}

Next, we observe that $\check{G}$ in (2.13) has an eigenvector with zero eigenvalue. Indeed, let us consider

$$
\iota_{k} \breve{G}=2 k^{i} G_{i j} \star d X^{j}-2 \star \mathrm{k}+\frac{2}{|k|^{2}}\left(\iota_{k} \mathrm{v}\right) \star \mathrm{v}=0,
$$

which vanishes due to (2.9) . In a similar way, for the field strength $\check{H}$ we find after a short computation that

$$
\iota_{k} \check{H}=0 .
$$

Thus, even though the original $D$-dimensional metric $G_{i j}$ is non-degenerate, the matrix $\check{G}_{i j}$ defined via (2.13) has one vanishing eigenvalue with eigenvector $k$.

\footnotetext{
${ }^{1}$ Our convention is that the symmetrization and anti-symmetrization of indices contains a factor of $1 / n$ !.
} 
Moreover, it turns out that $k$ is also a Killing vector for $\check{G}$ (and similarly for $\check{H}$ ), that is

$$
\mathcal{L}_{k} \check{G}=0, \quad \mathcal{L}_{k} \check{H}=0 .
$$

Given that $k$ is a null-vector, we can perform a change of coordinates. Assuming without loss of generality that $k^{1}$ is non-zero, we transform the matrix $\breve{G}$ as

$$
\check{\mathcal{G}}_{i j}=\left(\mathcal{T}^{T} \check{G} \mathcal{T}\right)_{i j}, \quad \quad \mathcal{T}^{i}{ }_{j}=\left(\begin{array}{c:c}
k^{1} & 0 \\
\hdashline k^{2} & \\
\vdots & \mathbb{1} \\
k^{D} &
\end{array}\right) .
$$

In the transformed matrix $\check{\mathcal{G}}_{i j}$ all entries along the $i, j=1$ direction vanish, and we therefore arrive at the expression

$$
\check{\mathcal{G}}_{i j}=\left(\begin{array}{c:c}
0 & 0 \\
\hdashline 0 & \check{G}_{a b} \\
\hdashline
\end{array}\right)
$$

where $a, b=2, \ldots, D$. Turning to the field strength $\check{H}$ and employing the matrix $\mathcal{T}^{i}{ }_{j}$, we transform $\breve{H}$ as follows

$$
\check{\mathcal{H}}_{i j k}=\check{H}_{l m n} \mathcal{T}_{i}^{l} \mathcal{T}^{m}{ }_{j} \mathcal{T}^{n}{ }_{k}
$$

Similarly to the transformed metric $\check{\mathcal{G}}_{i j}$, we again find that all components of $\check{\mathcal{H}}$ along the $i=1$ direction vanish, that is

$$
\check{\mathcal{H}}_{1 j k}=0 .
$$

From (2.19) and (2.21) we can conclude that in the action (2.12) the forms corresponding to the $i=1$ direction have dropped out.

\section{Change of coordinates}

However, this observation does not imply that we have arrived at a lower-dimensional theory. In particular, let us consider the transformed basis of one-forms given by $e^{i}=\left(\mathcal{T}^{-1}\right)^{i}{ }_{j} d X^{j}$. For the transformation matrix $\mathcal{T}$ shown in (2.18) we find

$$
e^{1}=\frac{1}{k^{1}} d X^{1}, \quad e^{a}=d x^{a}-\frac{k^{a}}{k^{1}} d X^{1}
$$


where again $a=2, \ldots, D$. Note that the algebra of one-forms $\left\{e^{a}\right\}$ in general does not close, but requires the basis of forms in the full $D$-dimensional space. More concretely, we have

$$
d e^{a}=\frac{1}{k^{1}}\left(k^{1} \partial_{b} k^{a}-k^{a} \partial_{b} k^{1}\right) e^{1} \wedge e^{b} .
$$

Therefore, in order for $\left\{e^{a}\right\}$ to close on itself and to properly reduce the $D$ dimensional target space to $(D-1)$ dimensions, for all $a, b \in\{2, \ldots, D\}$ we have to require

$$
0=k^{1} \partial_{b} k^{a}-k^{a} \partial_{b} k^{1}
$$

Performing a change of coordinates in the original geometry, it is always possible to choose $k^{a}=0$ for all $a=2, \ldots, D$. The requirement (2.24) can therefore be satisfied. Furthermore, using (2.17) and (2.6) together with $k^{a}=0$ we can show that the components $\check{G}_{a b}$ and $\check{H}_{a b c}$ do not depend on the coordinates in the direction $k$. Hence, after this change of coordinates we have arrived at a $(D-1)$ dimensional target-space background.

\subsection{Generalization to multiple isometries}

In the last two sections, we have described how to reduce the number of targetspace dimensions of a non-linear sigma-model by gauging a single world-sheet symmetry. In this section, following [14], we generalize this procedure to multiple, possibly non-abelian, symmetries.

\section{Original action and isometries}

We start again from the ungauged sigma-model action shown in equation (2.1), and assume that this action is invariant under the global symmetries

$$
\delta_{\epsilon} X^{i}=\epsilon^{\alpha} k_{\alpha}^{i}(X),
$$

labeled by $\alpha=1, \ldots, N$. We can assume without loss of generality that the vectors $k_{\alpha}$ are linearly independent. Furthermore, in order for the algebra of variations to close, the algebra generated by the vectors $k_{\alpha}$ is required to close,

$$
\left[k_{\alpha}, k_{\beta}\right]_{\mathrm{L}}=f_{\alpha \beta}^{\gamma} k_{\gamma} \text {. }
$$

Demanding then the action (2.1) to be invariant under (2.25) translates into the following conditions, generalizing the expressions given in section 2.1

$$
\mathcal{L}_{k_{\alpha}} G=0, \quad \iota_{k_{\alpha}} H=d \mathrm{v}_{\alpha}, \quad \mathcal{L}_{k_{\alpha}} \Phi=0 .
$$

The one-forms $v_{\alpha}$ are defined only up to a closed part, and their dual vector fields will be denoted by $v_{\alpha}$ in the following. 


\section{Gauged action}

Next, we gauge the symmetries (2.25). The resulting action has been derived in [83,84] and reads (in the notation of [14]) as

$$
\begin{aligned}
\widehat{\mathcal{S}}= & -\frac{1}{2 \pi \alpha^{\prime}} \int_{\partial \Sigma}\left[\frac{1}{2} G_{i j}\left(d X^{i}+k_{\alpha}^{i} A^{\alpha}\right) \wedge \star\left(d X^{j}+k_{\beta}^{j} A^{\beta}\right)+\frac{\alpha^{\prime}}{2} R \Phi \star 1\right] \\
& -\frac{i}{2 \pi \alpha^{\prime}} \int_{\Sigma} \frac{1}{3 !} H_{i j k} d X^{i} \wedge d X^{j} \wedge d X^{k} \\
& -\frac{i}{2 \pi \alpha^{\prime}} \int_{\partial \Sigma}\left[\mathrm{v}_{\alpha} \wedge A^{\alpha}+\frac{1}{2}\left(\iota_{\left.k_{[\underline{\alpha}} \mathrm{v}_{\underline{\beta}}\right]}\right) A^{\alpha} \wedge A^{\beta}\right] .
\end{aligned}
$$

The local symmetry transformations take the form

$$
\hat{\delta}_{\epsilon} X^{i}=\epsilon^{\alpha} k_{\alpha}^{i}, \quad \hat{\delta}_{\epsilon} A^{\alpha}=-d \epsilon^{\alpha}-\epsilon^{\beta} A^{\gamma} f_{\beta \gamma}{ }^{\alpha},
$$

and for invariance of the gauged action (2.28) under the transformations (2.29) we have to require that

$$
\mathcal{L}_{k_{[\underline{[}}} \mathrm{v}_{\underline{\beta}]}=f_{\alpha \beta} \mathrm{v}_{\gamma}, \quad \quad \iota_{k_{(\bar{\alpha}} \mathrm{v}_{\bar{\beta})}}=0 .
$$

Finally, we mention that by introducing the gauge fields $A^{\alpha}$, we again have enlarged the number of degrees of freedom of the theory. In order for the gauged action to be equivalent to the ungauged one, we demand the field strengths $F^{\alpha}$ to vanish, that is

$$
0=F^{\alpha}=d A^{\alpha}-\frac{1}{2} f_{\beta \gamma}^{\alpha} A^{\beta} \wedge A^{\gamma}
$$

\section{Integrating-out the gauge field}

The next step is to integrate-out the gauge field. The equations of motion for $A^{\alpha}$ read

$$
A^{\alpha}=-\left(\mathcal{M}^{-1}\right)^{\alpha \beta}\left(\mathbb{1}+i \star \mathcal{D} \mathcal{G}^{-1}\right)_{\beta}^{\gamma}(\mathrm{k}+i \star \mathrm{v})_{\gamma}
$$

where we remind the reader that $\alpha, \beta, \gamma=1, \ldots, N$ label the isometries which have been gauged. In the above expression, we have employed the notation

$$
\begin{array}{lll}
\mathcal{G}_{\alpha \beta} & =k_{\alpha}^{i} G_{i j} k_{\beta}^{j}, & \\
\mathcal{D}_{\alpha \beta}=\iota_{k_{\underline{\alpha}}} v_{\underline{\beta}]}, & \mathrm{k}_{\alpha}=k_{\alpha}^{i} G_{i j} d X^{j}, \\
\mathcal{M}_{\alpha \beta}=\left(\mathcal{G}-\mathcal{D} \mathcal{G}^{-1} \mathcal{D}\right)_{\alpha \beta}, &
\end{array}
$$


and have assumed the matrices $\mathcal{G}$ and $\mathcal{M}$ to be invertible,

$$
\operatorname{det} \mathcal{G} \neq 0, \quad \operatorname{det} \mathcal{M} \neq 0 .
$$

In the case of a single Killing vector this corresponds to the usual requirement that $|k|^{2} \neq 0$. After integrating-out the gauge field, the resulting action takes the general form shown in (2.12). Using matrix multiplication and suppressing the indices $\alpha, \beta, \ldots$ the metric $\breve{G}$ and field strength $\breve{H}$ are given by

$$
\begin{aligned}
& \check{G}=G+\left(\begin{array}{l}
\mathrm{k} \\
\mathrm{v}
\end{array}\right)^{T}\left(\begin{array}{cc}
-\mathcal{M}^{-1} & -\mathcal{M}^{-1} \mathcal{D G}^{-1} \\
+\mathcal{M}^{-1} \mathcal{D} \mathcal{G}^{-1} & +\mathcal{M}^{-1}
\end{array}\right) \wedge \star\left(\begin{array}{l}
\mathrm{k} \\
\mathrm{v}
\end{array}\right), \\
& \check{H}=H+\frac{1}{2} d\left[\left(\begin{array}{l}
\mathrm{k} \\
\mathrm{v}
\end{array}\right)^{T}\left(\begin{array}{cc}
+\mathcal{M}^{-1} \mathcal{D} \mathcal{G}^{-1} & +\mathcal{M}^{-1} \\
-\mathcal{M}^{-1} & -\mathcal{M}^{-1} \mathcal{D} \mathcal{G}^{-1}
\end{array}\right) \wedge\left(\begin{array}{l}
\mathrm{k} \\
\mathrm{v}
\end{array}\right)\right] .
\end{aligned}
$$

Furthermore, we note that the requirement of vanishing field strength shown in equation (2.31) imposes additional constraints. These are the generalizations of (2.14) which read

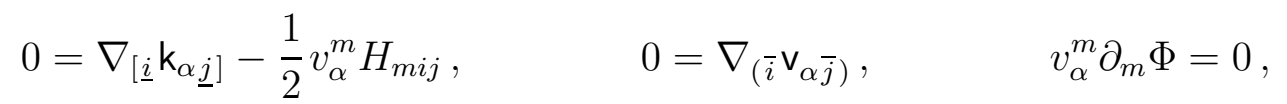

where $v_{\alpha}=\mathrm{v}_{\alpha i} G^{i j} \partial_{j}$ are the vector fields dual to the one-forms $\mathrm{v}_{\alpha}$. Details on the derivation of (2.36) can be found in appendix A. Moreover, employing (2.27) and (2.30) we can determine the following algebra

$$
\left[k_{\alpha}, k_{\beta}\right]_{\mathrm{L}}=f_{\alpha \beta}^{\gamma} k_{\gamma}, \quad\left[k_{\alpha}, v_{\beta}\right]_{\mathrm{L}}=f_{\alpha \beta}^{\gamma} v_{\gamma}, \quad\left[v_{\alpha}, v_{\beta}\right]_{\mathrm{L}}=f_{\alpha \beta}{ }^{\gamma} k_{\gamma} .
$$

\section{Reduced geometry}

Next, we observe that, as before, the vectors $k_{\alpha}$ are null-vectors as well as Killing vectors for $\check{G}$ and $\check{H}$. In particular, we have

$$
\begin{array}{llrl}
\iota_{k_{\alpha}} \check{G}=0, & \mathcal{L}_{k_{\alpha}} \check{G}=0, \\
\iota_{k_{\alpha}} \check{H}=0, & \mathcal{L}_{k_{\alpha}} \check{H}=0 .
\end{array}
$$

We can therefore perform a change of basis in the following way

$$
\check{\mathcal{G}}_{i j}=\left(\mathcal{T}^{T} \check{G} \mathcal{T}\right)_{i j}, \quad \mathcal{T}^{i}{ }_{j}=\left(\begin{array}{ccc:c}
k_{1}^{1} & \cdots & k_{N}^{1} & \\
\vdots & & \vdots & 0 \\
k_{1}^{N} & \cdots & k_{N}^{N} & \\
\hdashline \vdots & & \vdots & \\
\hdashline k_{1}^{D} & \cdots & k_{N}^{D} & \mathbb{1}
\end{array}\right),
$$


where we arranged the matrix $\mathcal{T}$ in such a way that the upper-left block is invertible. (This is always possible provided the Killing vectors are linearly independent.) The schematic form of the transformed matrix $\breve{\mathcal{G}}$ is

$$
\check{\mathcal{G}}_{i j}=\left(\begin{array}{c:c}
0 & 0 \\
\hdashline 0 & \check{G}_{a b}
\end{array}\right),
$$

where $a, b=(N+1), \ldots, D$. Similarly, for the field strength we consider the transformation $\check{\mathcal{H}}_{i j k}=\check{H}_{l m n} \mathcal{T}_{i}^{l} \mathcal{T}^{m}{ }_{j} \mathcal{T}^{n}{ }_{k}$, for which we find

$$
\check{\mathcal{H}}_{1 i j}=0, \quad \check{\mathcal{H}}_{2 i j}=0, \quad \ldots \quad \check{\mathcal{H}}_{N i j}=0 .
$$

Performing furthermore a change of coordinates such that $k_{\alpha}^{i}=0$ for $i=N+$ $1, \ldots, D$ and using (2.38), we can show that the algebra of transformed one-forms $\left\{e^{a}\right\}$ closes under $d$, and that components $\check{G}_{a b}$ and $\check{H}_{a b c}$ do not depend on $X^{i}$ for $i=1, \ldots, N$. Hence, we have arrived at sigma-model whose target-space geometry is reduced by $N$ dimensions.

\subsection{Summary and discussion}

\section{Summary}

Let us briefly summarize and discuss the results obtained in this section.

- Our starting point was the non-linear sigma-model action (2.1) which we assumed to be invariant under global transformations $\delta_{\epsilon} X^{i}=\epsilon^{\alpha} k_{\alpha}^{i}$ with $\alpha=1, \ldots, N$. This requirement implies that the vectors $k_{\alpha}$ have to be Killing, it gave rise to the definition of one-forms $\mathrm{v}_{\alpha}$, and it implied that $k_{\alpha}^{m} \partial_{m} \Phi=0$. In formulas this reads

$$
\mathcal{L}_{k_{\alpha}} G=0, \quad \iota_{k_{\alpha}} H=d \mathrm{v}_{\alpha}, \quad \mathcal{L}_{k_{\alpha}} \Phi=0 .
$$

Note furthermore that the algebra of Killing vectors can be non-abelian with structure constants $f_{\alpha \beta}{ }^{\gamma}$.

- Next, we gauged this world-sheet symmetry. The gauged action has been shown in (2.28), and for gauge invariance one has to require (2.30), that is

$$
\mathcal{L}_{k_{[\underline{\alpha}} \underline{\left.\mathrm{\beta}^{\prime}\right]}}=f_{\alpha \beta} \mathrm{v}_{\gamma}, \quad \iota_{k_{(\bar{\alpha}}} \mathrm{v}_{\bar{\beta})}=0 .
$$


- Finally, through the gauging procedure gauge fields $A^{\alpha}$ have been introduced. In order to for the gauged and ungauged theory to be equivalent, we required that the corresponding field strengths vanish, $F^{\alpha}=0$. The latter condition is satisfied provided that

$$
\mathcal{L}_{v_{\alpha}} G=0, \quad \iota_{v_{\alpha}} H=d \mathrm{k}_{\alpha}, \quad \mathcal{L}_{v_{\alpha}} \Phi=0 .
$$

If these conditions are met, it is possible to gauge the isometries associated to $k_{\alpha}$ without introducing additional degrees of freedom. Integrating-out the gauge field from the action and performing a change of coordinates gives a target-space background, which is reduced by $N$ dimensions. The expressions for the reduced metric and field strength are obtained from (2.35) using the change of coordinates shown in (2.39).

\section{Remarks}

Let us conclude this section with the following remarks:

- We observe that the conditions (2.42) and (2.44) are the same expressions with $k_{\alpha}$ and $v_{\alpha}$ interchanged. Writing them in a coordinate-dependent way and after a slight rearrangement, we have

$$
\begin{array}{lll}
0=\nabla_{(\bar{i}} \mathrm{k}_{\alpha \bar{j})}, & 0=\nabla_{[\underline{i}} \mathrm{k}_{\alpha \underline{j}]}-\frac{1}{2} v_{\alpha}^{m} H_{m i j}, & 0=k_{\alpha}^{m} \partial_{m} \Phi, \\
0=\nabla_{(\bar{i}} \mathrm{v}_{\alpha \bar{j})}, & 0=\nabla_{\left[\underline{i} \mathbf{v}_{\alpha \underline{j}]}\right.}-\frac{1}{2} k_{\alpha}^{m} H_{m i j}, & 0=v_{\alpha}^{m} \partial_{m} \Phi,
\end{array}
$$

together with

$$
\begin{array}{ll} 
& {\left[k_{\alpha}, k_{\beta}\right]_{\mathrm{L}}=f_{\alpha \beta}{ }^{\gamma} k_{\gamma},} \\
& {\left[k_{\alpha}, v_{\beta}\right]_{\mathrm{L}}=f_{\alpha \beta}{ }^{\gamma} v_{\gamma},} \\
& {\left[v_{\alpha}, v_{\beta}\right]_{\mathrm{L}}=f_{\alpha \beta}{ }^{\gamma} k_{\gamma} .}
\end{array}
$$

- We also remark that for vanishing $H$-flux, the components $k_{\alpha}^{i}$ and $v_{\alpha}^{i}$ have to be covariantly constant. Hence the structure constants of the isometry algebra vanish,

$$
H=0 \quad \Longrightarrow \quad \begin{aligned}
& 0=\nabla_{i} k_{\alpha}^{j} \\
& 0=\nabla_{i} v_{\alpha}^{j}
\end{aligned} \quad \Longrightarrow \quad f_{\alpha \beta}^{\gamma}=0 .
$$

Furthermore, for $H=0$ the one-forms $\mathrm{v}_{\alpha}$, and consequently the vector fields $v_{\alpha}$, can all be chosen to be zero. 


\section{Relation to Roček \& Verlinde}

In this section we make contact between our discussion in section 2 and the work of Roček and Verlinde (RV) [6], which has also been used in [52]. In particular, we assume the metric $G$ and the field strength $H$ appearing in the world-sheet action (2.1) to have a specific form, corresponding to torus fibrations over a base manifold. These are the geometries relevant for double field theory.

\subsection{Single doubled dimension}

Let us start by applying the formalism explained in the previous section to the setting of Roček and Verlinde [6].

\section{Background}

More specifically, the RV background is given by first considering the following two-dimensional non-linear sigma-model

$$
\mathcal{S}^{\prime}=\frac{1}{2 \pi \alpha^{\prime}} \int d z d \bar{z}\left(\left[g_{a b}(X)+b_{a b}(X)\right] \partial X^{a} \bar{\partial} X^{b}+\frac{\alpha^{\prime}}{4} R \phi(X)\right),
$$

where $a, b=3, \ldots, D+1$. Note that here we employed a complex basis on the world-sheet. In this action, the target space is $(D-1)$-dimensional with coordinates $X^{a}$. Next, we enlarge the target space by introducing two additional coordinates $X_{L}$ and $X_{R}$, and define a new $(D+1)$-dimensional parent sigma-model as

$$
\begin{aligned}
\mathcal{S}_{\mathrm{LR}}=\mathcal{S}^{\prime}+\frac{1}{2 \pi \alpha^{\prime}} \int d z d \bar{z}\left[\partial X_{L} \bar{\partial} X_{L}+\partial X_{R} \bar{\partial} X_{R}+2 B(X) \partial X_{R} \bar{\partial} X_{L}\right. \\
\left.+2 G_{a}^{L}(X) \partial X^{a} \bar{\partial} X_{L}+2 G_{a}^{R}(X) \bar{\partial} X^{a} \partial X_{R}\right]
\end{aligned}
$$

whose couplings $B(X), G_{a}^{L}(X)$ and $G_{a}^{R}(X)$ depend only on the $(D-1)$ coordinates $X^{a}$. Note that this action is consistent with the action (2.1). By comparison with the general form of a sigma-model, we see that (3.2) determines a $(D+1)$ dimensional metric and $B$-field as follows

$$
G_{I J}=\left(\begin{array}{ccc}
1 & B & G_{b}^{L} \\
B & 1 & G_{b}^{R} \\
G_{a}^{L} & G_{a}^{R} & g_{a b}
\end{array}\right), \quad B_{I J}=\left(\begin{array}{ccc}
0 & -B & -G_{b}^{L} \\
+B & 0 & +G_{b}^{R} \\
+G_{a}^{L} & -G_{a}^{R} & b_{a b}
\end{array}\right)
$$

We then perform the field redefinitions

$$
X^{1}=X_{L}+X_{R}, \quad X^{2}=X_{L}-X_{R}
$$


and $G_{a}^{ \pm}=G_{a}^{L} \pm G_{a}^{R}$, after which the parent background (3.3) can be expressed as

$$
G_{I J}=\left(\begin{array}{ccc}
\frac{1+B}{2} & 0 & \frac{1}{2} G_{b}^{+} \\
0 & \frac{1-B}{2} & \frac{1}{2} G_{b}^{-} \\
\frac{1}{2} G_{a}^{+} & \frac{1}{2} G_{a}^{-} & g_{a b}
\end{array}\right), \quad B_{I J}=\left(\begin{array}{ccc}
0 & +\frac{1}{2} B & -\frac{1}{2} G_{b}^{-} \\
-\frac{1}{2} B & 0 & -\frac{1}{2} G_{b}^{+} \\
+\frac{1}{2} G_{a}^{-} & +\frac{1}{2} G_{a}^{+} & b_{a b}
\end{array}\right) .
$$

We also mention that compared to the previous section, we have slightly changed our notation for the indices. In the following we use

$$
I, J=1, \ldots, D+1, \quad i, j=2, \ldots, D+1, \quad a, b=3, \ldots, D+1 .
$$

\section{Isometries}

In [6], and also here, the quantities $B, G_{a}^{ \pm}, g_{a b}$ and $b_{a b}$ only depend on the coordinates $X^{a}$. Therefore, the metric $G_{I J}$ in (3.5) has at least two abelian isometries generated by the Killing vectors

$$
k_{(1)}^{I}=\left(\begin{array}{l}
1 \\
0 \\
0
\end{array}\right) \quad \text { and } \quad k_{(2)}^{I}=\left(\begin{array}{l}
0 \\
1 \\
0
\end{array}\right) .
$$

Note that $G_{I J}$ and $B_{I J}$ do not constitute the most general $(D+1)$-dimensional sigma-model background, but are of rather restricted form. For a general background with two abelian isometries of the form (3.7), the sigma-model action would be invariant under $O(2) \times O(2)$ transformations. However, in order to preserve the restricted choice of the background as given in equations (3.3) and (3.5), the action is only invariant under a $\mathbb{Z}_{2}$ transformation. This can be formulated as an automorphism

$$
X_{L} \longrightarrow \tilde{X}_{L}=+X_{L}, \quad X_{R} \longrightarrow \tilde{X}_{R}=-X_{R},
$$

or in terms of the coordinates (3.4) as

$$
X^{1}=X_{L}+X_{R} \quad \longleftrightarrow \quad X^{2}=X_{L}-X_{R}
$$

together will the following transformation on the background fields:

$$
B \longleftrightarrow-B, \quad G_{a}^{ \pm} \longleftrightarrow G_{a}^{\mp} .
$$

As we will see below, for the reduced background this transformation is nothing else then standard T-duality. 


\section{Reduced background}

Let us now follow the procedure explained in the previous section. In particular, we gauge a symmetry of the sigma-model by the following linear combination of the isometries (3.7)

$$
k^{I}=\left(\begin{array}{l}
\mathrm{a} \\
\mathrm{b} \\
0
\end{array}\right), \quad \mathrm{a}^{2}+\mathrm{b}^{2} \neq 0 .
$$

Next, we determine the one-form $v$ defined via (2.4). Solving the constraints (2.14), we obtain

$$
\mathrm{v}=\frac{\mathrm{a}}{2}\left[(1-B) d X^{2}+G_{\alpha}^{-} d X^{\alpha}\right]+\frac{\mathrm{b}}{2}\left[(1+B) d X^{1}+G_{\alpha}^{+} d X^{\alpha}\right] .
$$

However, imposing furthermore the condition (2.9) leads to $a b=0$ which leaves only the two cases

$$
(a \neq 0, b=0), \quad(a=0, b \neq 0) .
$$

Note that here and in the following we assume that the field strength $H=d B$ is non-zero along the directions $I=1,2$, that is $H_{12 a} \neq 0$. For vanishing $H$, the restriction (3.13) does not apply.

With the above data, we can now determine the $(D+1)$-dimensional matrix $\check{G}$ defined in (2.13). Since this matrix has a null-eigenvector, we perform a change of coordinates such that the reduced metric $\breve{\mathcal{G}}$ only depends on the following $D$ dimensional basis of the co-tangent space

$$
d Y^{2}=\mathrm{b} d X^{1}-\mathrm{a} d X^{2}, \quad d Y^{a}=d X^{a} .
$$

The dependence of the fields can be relabelled as $X^{a}=Y^{a}$. With $i, j=2, \ldots, D+1$ we determine the components of the reduced metric as

$$
\check{\mathcal{G}}_{i j}=\left(\begin{array}{cc}
\frac{1 \mp B}{1 \pm B} & -\frac{G_{b}^{\mp}}{1 \pm B} \\
-\frac{G_{a}^{\mp}}{1 \pm B} & g_{a b} \mp \frac{G_{a}^{+} G_{b}^{+}-G_{a}^{-} G_{b}^{-}}{2(1 \pm B)}
\end{array}\right),
$$

where the upper sign corresponds to the first choice in (3.13) and the lower sign to the second. Performing the same procedure for the $H$-field $\check{H}$ given in (2.13), we can infer the corresponding reduced $B$-field as

$$
\check{\mathcal{B}}_{i j}=\left(\begin{array}{cc}
0 & \pm \frac{1}{1 \pm B} G_{b}^{ \pm} \\
\mp \frac{1}{1 \pm B} G_{a}^{ \pm} & b_{a b} \mp \frac{G_{a}^{-} G_{b}^{+}-G_{a}^{+} G_{b}^{-}}{2(1 \pm B)}
\end{array}\right) .
$$


Since these two theories descend from a single parent theory, in the following we call them child theories. As it is well-known, the two backgrounds specified by the upper and lower sign are T-dual to each other. The transformations between them is given by the Buscher rules [4,5, 3].

\section{Discussion}

In this subsection, we have related the results of Roček and Verlinde in [6] to our discussion in section 2, We made the following observations:

- First, for the choice of metric and $B$-field shown in (3.3), we have reproduced the two reduced backgrounds of [6] specified in (3.15) and (3.16).

- Second, since the parent background has the two abelian isometries (3.7), one might have expected that any linear combination (3.11) can be gauged. This would give rise to continuous family of reduced backgrounds. However, for non-vanishing $H$-flux along the $I=1$ and $I=2$ direction the constraints (2.45) allow only for two solutions, which explains the results of [6] in a broader context.

- Third, for the RV background we saw that at the level of the parent theory, T-duality corresponds to two different choices of Killing vectors. In view of our discussion in section 2, this means choosing either $k$ or $v$ for the gauging procedure.

\subsection{Conformal invariance of parent and child theories}

In this section we compare the $\beta$-functions of the $(D+1)$-dimensional parent theory with the $\beta$-functions of the $D$-dimensional child theory. In general the $\beta$-functions are given by the following set of equations

$$
\begin{aligned}
& \beta_{I J}^{G}=\alpha^{\prime} R_{I J}+2 \alpha^{\prime} \nabla_{I} \nabla_{J} \Phi-\frac{\alpha^{\prime}}{4} H_{I K L} H_{J}{ }^{K L}+\mathcal{O}\left(\alpha^{\prime 2}\right) \\
& \beta_{I J}^{B}=-\frac{\alpha^{\prime}}{2} \nabla^{K} H_{K I J}+\alpha^{\prime} \nabla^{K} \Phi H_{K I J}+\mathcal{O}\left(\alpha^{\prime 2}\right) \\
& \beta^{\Phi}=\frac{D+1-D_{\text {crit }}}{4}-\frac{\alpha^{\prime}}{2} \nabla^{2} \Phi+\alpha^{\prime} \nabla^{K} \Phi \nabla_{K} \Phi-\frac{\alpha^{\prime}}{24} H_{I J K} H^{I J K}+\mathcal{O}\left(\alpha^{\prime 2}\right) .
\end{aligned}
$$

\section{$\beta$-functionals for a three-dimensional parent theory}

In order to keep our discussion tractable we focus on $D=2$, that is we consider a three-dimensional parent theory. In this case the $H$-field has only one non-trivial 
component, namely $H_{123}=\frac{1}{2} \partial_{3} B\left(X^{3}\right)=\frac{1}{2} B^{\prime}$. The vanishing of the $\beta$-functional for $B$ then reduces to

$$
0=H_{123}^{\prime}-\Gamma_{3 \mu}^{\mu} H_{123}-2 \Phi^{\prime} H_{123}, \quad \partial_{1} \Phi=\partial_{2} \Phi=0 .
$$

Note that here and in the remainder of this section, we denote the derivative with respect to $X^{3}$ by a prime. Since $\Gamma_{3 \mu}^{\mu}=\frac{1}{2} \log (\operatorname{det} G)^{\prime}$, it follows by integration that the dilaton is given by

$$
\Phi\left(X^{3}\right)=\phi_{0}+\frac{1}{4} \log \left[\frac{\left(H_{123}\right)^{2}}{\operatorname{det} G}\right]
$$

with $\phi_{0}$ a constant. For this solution of the dilaton, all $\beta^{B}$ - and $\beta^{G}$-functionals in three dimensions are vanishing, except

$$
\beta_{33}^{G}=\alpha^{\prime}\left[2 \Phi^{\prime \prime}-\frac{(\operatorname{det} G)^{\prime}}{\operatorname{det} G} \Phi^{\prime}\right] .
$$

Let us now combine equation (3.19) and the requirement of vanishing $\beta$-functional (3.20). We can infer two classes of solutions:

a) The first class of models is characterized by a constant dilaton $\Phi$, which trivially satisfy $\beta_{33}^{G}=0$. The flat torus in three dimensions as well as the $S U(2)$ and $S L(2, \mathbb{R})$ models are examples thereof. Note also that a constant dilaton implies

$$
\frac{H_{123}}{\sqrt{\operatorname{det} G}}=\frac{\frac{1}{2} B^{\prime}}{\sqrt{\operatorname{det} G}}=\gamma_{1}=\text { const. }
$$

b) The second class of models is given by a non-constant dilaton. The vanishing of $\beta_{33}^{G}$ then implies that

$$
\frac{\Phi^{\prime}}{\sqrt{\operatorname{det} G}}=\gamma_{2}=\text { const. } \neq 0
$$

We also mention that for the class of solutions a), the $\beta^{\Phi}$-functional (up to linear order in $\alpha^{\prime}$ ) is constant. Similarly, one can show in a somewhat more involved way that $\beta^{\Phi}$ is constant also for case $\mathrm{b}$ ).

\section{$\beta$-functionals for the two-dimensional child theories}

Let us now turn to the two-dimensional child theories. The metric and $B$-field are given by the expressions shown in (3.15) and (3.16), and the dilaton of the child theories $\check{\Phi}$ is obtained by the usual Buscher shift, namely

$$
\check{\Phi}=\Phi-\frac{1}{2} \log (1 \pm B) \text {. }
$$


For the $\beta$-functionals of the child theories we observe that in two-dimensions the $H$-field is vanishing, and hence the $\beta^{B}$-functional vanishes identically,

$$
\check{\beta}_{i j}^{B}=0 .
$$

For $\check{\beta}^{G}$ we can perform an explicit computation using for instance a computer algebra program. With the dilaton of the parent theory given in (3.19), we find that the $\beta^{G}$-functional of the child theories vanish, that is

$$
\check{\beta}_{i j}^{G}=0 .
$$

The $\beta^{\Phi}$-functional for the child theories are computed slightly differently for the cases a) and b) mentioned above. However, for both we find that $\check{\beta}^{\Phi}$ is constant. Moreover, the contribution $\Delta^{(1)}$ at linear order in $\alpha^{\prime}$ is the same for the parent and child theories, i.e.

$$
\begin{aligned}
& \beta^{\Phi}=\frac{D+1-D_{\text {crit }}}{4}+\alpha^{\prime} \Delta^{(1)}+\mathcal{O}\left(\alpha^{2}\right), \\
& \check{\beta}^{\Phi}=\frac{D-D_{\text {crit }}}{4}+\alpha^{\prime} \Delta^{(1)}+\mathcal{O}\left(\alpha^{2}\right) .
\end{aligned}
$$

Note that the tree-level contribution to this $\beta$-functional is different for the parent and child theories. In view of their applications to T-duality, we require the child theories to be string theories, that is $D=D_{\text {crit }}$. In turn, this implies that the $\beta^{\Phi}$-functional of the parent theory is non-vanishing and hence the parent theory is not a string theory.

\subsection{Multiple doubled dimensions}

Let us now generalize our discussion from section 3.1 to multiple doubled dimensions. Here, we focus on the abelian case; the non-abelian situation will be discussed elsewhere.

\section{Doubled background}

We begin by specifying the geometry of the doubled background. We start again from the sigma-model (3.1), and enlarge the target space by $2 N$ coordinates $\left\{X_{L}^{\alpha}, X_{R}^{\alpha}\right\}$. The resulting action reads

$$
\begin{aligned}
\mathcal{S}_{\mathrm{LR}}=\mathcal{S}^{\prime}+\frac{1}{2 \pi \alpha^{\prime}} \int d z d \bar{z}\left[\delta_{\alpha \beta} \partial X_{L}^{\alpha} \bar{\partial} X_{L}^{\beta}\right. & +\delta_{\alpha \beta} \partial X_{R}^{\alpha} \bar{\partial} X_{R}^{\beta}+2 B_{\alpha \beta} \partial X_{R}^{\alpha} \bar{\partial} X_{L}^{\beta} \\
& \left.+2 G_{a \beta}^{L} \partial X^{a} \bar{\partial} X_{L}^{\beta}+2 G_{a \beta}^{R} \bar{\partial} X^{a} \partial X_{R}^{\beta}\right]
\end{aligned}
$$


where $\alpha, \beta=1, \ldots, N$ and $a, b=2 N+1, \ldots, 2 N+D$, and where we assume all couplings $B_{\alpha \beta}, G_{a \beta}^{L / R}$ to only depend on the coordinates $X^{a}$. Next, we perform a change of coordinates

$$
X^{\alpha}=X_{L}^{\alpha}+X_{R}^{\alpha}, \quad X^{N+\alpha}=X_{L}^{\alpha}-X_{R}^{\alpha}
$$

and define

$$
\begin{array}{ll}
B_{\alpha \beta}^{+}=\frac{1}{2}\left(B_{\alpha \beta}+B_{\alpha \beta}^{T}\right), & G_{a \beta}^{+}=G_{a \beta}^{L}+G_{a \beta}^{R}, \\
B_{\alpha \beta}^{-}=\frac{1}{2}\left(B_{\alpha \beta}-B_{\alpha \beta}^{T}\right), & G_{a \beta}^{-}=G_{a \beta}^{L}-G_{a \beta}^{R} .
\end{array}
$$

Suppressing the indices, the resulting metric and $B$-field (written in a basis $X^{I}=$ $\left.\left\{X^{\alpha}, X^{N+\alpha}, X^{a}\right\}\right)$ take the following form

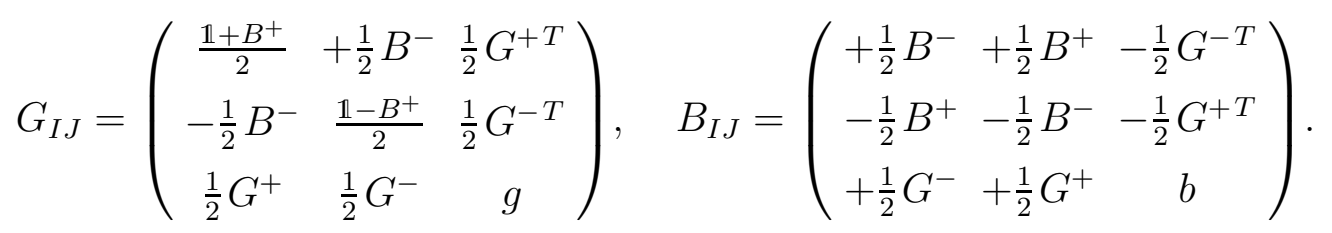

Note that since all components only depend on $X^{a}$, this background has at least $2 N$ isometries. Without loss of generality, we can express the corresponding Killing vectors as

$$
k_{\alpha}^{I}=\left(\begin{array}{c}
\vdots \\
1 \\
\vdots \\
\hdashline \\
0 \\
-0
\end{array}\right)=\delta_{\alpha}^{I}, \quad v_{\alpha}^{I}=\left(\begin{array}{c}
0 \\
0 \\
\vdots \\
1 \\
\vdots \\
0
\end{array}\right)=\delta_{N+\alpha}^{I} .
$$

Furthermore, the background specified in (3.30) is invariant under the following combined transformation

$$
X^{\alpha} \longleftrightarrow X^{N+\alpha}, \quad G_{a \beta}^{+} \longleftrightarrow G_{a \beta}^{-}, \quad B_{\alpha \beta} \longleftrightarrow-B_{\alpha \beta},
$$

which implies $k_{\alpha} \leftrightarrow v_{\alpha}$ for the Killing vectors.

\section{Reduced background}

Let us now follow the procedure introduced in section 2 to obtain the reduced background. In particular, we want to gauge the $N$ isometries specified by $k_{\alpha}$ and integrate-out the corresponding gauge fields. 
The vectors (3.31) satisfy the conditions shown in (2.45) and (2.46). We can therefore apply the reduction procedure, for which the matrices $\breve{G}$ and $\check{B}$ defined in (2.35) take the general form

$$
\check{G}_{I J}=\left(\begin{array}{c:cc}
0 & 0 & 0 \\
\hdashline 0 & \check{G}^{(22)} & \check{G}^{(23)} \\
0 & \check{G}^{(32)} & \check{G}^{(33)}
\end{array}\right), \quad \quad \check{B}_{I J}=\left(\begin{array}{c:cc}
0 & 0 & 0 \\
\hdashline 0 & \check{B}^{(22)} & \check{B}^{(23)} \\
0 & \check{B}^{(32)} & \check{B}^{(33)}
\end{array}\right)
$$

where we applied a gauge transformation to remove a constant term in $\check{B}$. The explicit expressions for the components can easily be determined from (2.35). However, to illustrate the underlying structure we consider the case $B^{-}=0$ for which we find

$$
\begin{aligned}
& \check{G}^{(22)}=\mathbb{1}\left(1+B^{+}\right)^{-1}\left(1-B^{+}\right) \\
& \check{G}^{(23)}=+\mathbb{1}\left(1+B^{+}\right)^{-1} G^{-T}, \\
& \check{G}^{(33)}=g-\frac{1}{2} G^{+}\left(1+B^{+}\right)^{-1} G^{+T}+\frac{1}{2} G^{-}\left(1+B^{+}\right)^{-1} G^{-T} \\
& \check{B}^{(22)}=0 \\
& \check{B}^{(23)}=-\mathbb{1}\left(1+B^{+}\right)^{-1} G^{+T}, \\
& \check{B}^{(33)}=b-\frac{1}{2} G^{-}\left(1+B^{+}\right)^{-1} G^{+T}+\frac{1}{2} G^{+}\left(1+B^{+}\right)^{-1} G^{-T} .
\end{aligned}
$$

These are the generalizations of (3.15) and (3.16). Note that if we choose to perform the reduction by gauging the vectors $v_{\alpha}$ instead of $k_{\alpha}$, we obtain (3.34) with the replacements $B^{+} \rightarrow-B^{+}$and $G^{ \pm} \rightarrow G^{\mp}$. We have therefore shown, that our analysis of a single doubled dimensions generalizes to multiple doubled dimensions. In particular, T-duality is again given by interchanging the vector fields $k_{\alpha}$ and $v_{\alpha}$.

\section{Examples}

Let us now apply the procedure introduced in the previous sections to WessZumino-Witten models. We first consider the $S U(2)$ example, and then comment on the generalization to arbitrary Lie groups. Note that WZW models in the context of DFT have also been studied in [85, 86, , albeit in a rather different approach. 


\section{1 $S U(2)$ WZW model}

The $S U(2)$ WZW model corresponds to a conformal field theory on a three-sphere with non-vanishing $H$-flux. We therefore consider a three-dimensional parent theory and want to construct two-dimensional child theories.

\section{Parent theory}

We start by introducing the setting. We choose the following parametrization for the metric of a round three-sphere with radius $R$

$$
G_{I J}=R^{2}\left(\begin{array}{ccc}
\sin ^{2} \eta & 0 & 0 \\
0 & \cos ^{2} \eta & 0 \\
0 & 0 & 1
\end{array}\right) .
$$

The coordinates are given by $x^{I}=\left(\zeta_{1}, \zeta_{2}, \eta\right)$ with $\zeta_{1,2} \in[0,2 \pi)$ and $\eta \in[0, \pi / 2]$. In order for the three-dimensional model to be conformal, the $H$-flux and dilaton have to take the form

$$
H=2 R^{2} \sin \eta \cos \eta d \zeta_{1} \wedge d \zeta_{2} \wedge d \eta, \quad \Phi=\text { const. }
$$

And, indeed, for this background the one-loop $\beta$-functionals for the metric and $B$ field shown in (3.17) are vanishing. Note also that this background can be brought into the form (3.5). In particular, if we identify

$$
\begin{array}{lll}
X^{1}=R \zeta_{1}, & B=-\cos (2 \eta), & G_{3}^{+}=0, \\
X^{2}=R \zeta_{2}, & g_{33}=R^{2}, & G_{3}^{-}=0, \\
X^{3}=\eta, & &
\end{array}
$$

the metric shown in (3.5) becomes (4.1), and the field strength computed from $B_{I J}$ in (3.5) becomes (4.2).

\section{Child theories}

Next, this background has two abelian Killing vectors given by $k^{I}=(1,0,0)^{T}$ and $k^{I}=(0,1,0)^{T}$. In order to solve the constraints (2.45), we saw in section 3.1 that not every linear combination can be used for the reduction procedure but that only two choices are possible. In particular, because the metric and $B$-field of the three-sphere background can be brought into the RV form, (3.13) implies that the two allowed gaugings are

$$
k_{(1)}^{I}=\left(\begin{array}{l}
1 \\
0 \\
0
\end{array}\right), \quad k_{(2)}^{I}=\left(\begin{array}{l}
0 \\
1 \\
0
\end{array}\right) .
$$


We remark that the overall normalization of the Killing vectors drops out of all formulas. With the help of the identifications (4.3), we can now determine the child theories from the general expressions (3.15) and (3.16).

1. For the first Killing vector in (4.4), we obtain the following reduced background

$$
\check{\mathcal{G}}_{i j}=R^{2}\left(\begin{array}{cc}
\cot ^{2} \eta & 0 \\
0 & 1
\end{array}\right), \quad \quad \check{\mathcal{B}}_{i j}=0 .
$$

This geometry is known as the trumpet solution, which is a conformal model provided the dilaton is adjusted through the Buscher shift as

$$
\check{\Phi}=\Phi-\log (\sin \eta) \text {. }
$$

2. For the second Killing vector shown in equation (4.4), we obtain the reduced background as the cigar solution

$$
\check{\mathcal{G}}_{i j}=R^{2}\left(\begin{array}{cc}
\tan ^{2} \eta & 0 \\
0 & 1
\end{array}\right), \quad \quad \check{\mathcal{B}}_{i j}=0 .
$$

This is again a conformal model, if we adjust the reduced dilaton as

$$
\check{\Phi}=\Phi-\log (\cos \eta) \text {. }
$$

For the $S U(2)$ example we have therefore verified our above results, namely that for the RV-type background two isometries can be gauged. The resulting child theories are conformal, and it is well-known that they are T-dual to each other. In particular, T-duality corresponds to interchanging which Killing vector has been used to perform the reduction.

\subsection{General WZW model}

In this subsection we now comment on the generalization of the reduction procedure to arbitrary Lie groups. We first review some properties of Wess-ZuminoWitten models, and then discuss the gauging constraints (2.45) and (2.46).

\section{Action}

Let us start with a WZW model for a Lie group $G$. The corresponding action (without the dilaton term) is given by

$$
\begin{aligned}
\mathcal{S}= & \frac{1}{2 \pi \alpha^{\prime}} \int_{\partial \Sigma} \operatorname{Tr}\left[\frac{k}{4} g^{-1} d g \wedge \star g^{-1} d g\right] \\
& +\frac{i}{2 \pi \alpha^{\prime}} \int_{\Sigma} \operatorname{Tr}\left[\frac{k}{6} g^{-1} d g \wedge g^{-1} d g \wedge g^{-1} d g\right]
\end{aligned}
$$


where $g \in G$ and where $k$ denotes the level. The generators of the corresponding Lie algebra $\left\{t_{\mathrm{a}}\right\} \in \mathfrak{g}$ with $\mathrm{a}=1, \ldots, D$ are normalized such that

$$
\left[t_{\mathrm{a}}, t_{\mathrm{b}}\right]=i f_{\mathrm{ab}}{ }^{\mathrm{c}} t_{\mathrm{c}}, \quad \operatorname{Tr}\left(t_{\mathrm{a}} t_{\mathrm{b}}\right)=2 \delta_{\mathrm{ab}},
$$

and the left- and right-invariant forms are given by

$$
\omega_{L}=g^{-1} d g=\omega_{L}{ }^{a} t_{\mathrm{a}}, \quad \omega_{R}=d g g^{-1}=\omega_{R}{ }^{\mathrm{a}} t_{\mathrm{a}} .
$$

Using these conventions, we can determine the target-space metric $G$ and the field strength $H$ by comparing with (2.1) as follows

$$
\begin{aligned}
& G=-k \quad \delta_{\mathrm{ab}} \omega_{L}^{\mathrm{a}} \wedge \star_{L}^{\mathrm{b}}=-k \quad \delta_{\mathrm{ab}} \omega_{R}^{\mathrm{a}} \wedge \star \omega_{R}^{\mathrm{b}}, \\
& H=-\frac{i k}{3 !} f_{\mathrm{abc}} \omega_{L}^{\mathrm{a}} \wedge \omega_{L}^{\mathrm{b}} \wedge \omega_{L}^{\mathrm{c}}=-\frac{i k}{3 !} f_{\mathrm{abc}} \omega_{R}^{\mathrm{a}} \wedge \omega_{R}^{\mathrm{b}} \wedge \omega_{R}^{\mathrm{c}} .
\end{aligned}
$$

Here, $f_{\mathrm{abc}}=f_{\mathrm{ab}}{ }^{\mathrm{d}} \delta_{\mathrm{dc}}$ is completely anti-symmetric in its indices, and the minus sign in the metric ensures that $G_{\mathrm{ab}}$ is positive definite. Indeed, for $g$ unitary it follows that $\left(g^{-1} d g\right)^{\dagger}=-d^{-1} d g$ and thus the first line in (4.9) is negative definite.

\section{Geometry}

Let us now briefly recall some of the properties of the target-space geometry. First, the left- and right-invariant forms (4.11) satisfy the Maurer-Cartan equation, that is

$$
0=d \omega_{L}{ }^{\mathrm{a}}+\frac{i}{2} f_{\mathrm{bc}}{ }^{\mathrm{a}} \omega_{L}^{\mathrm{b}} \wedge \omega_{L}^{\mathrm{c}}, \quad 0=d \omega_{R}^{\mathrm{a}}-\frac{i}{2} f_{\mathrm{bc}}{ }^{\mathrm{a}} \omega_{R}{ }^{\mathrm{b}} \wedge \omega_{R}{ }^{\mathrm{c}} .
$$

Next, we choose a coordinate basis of one-forms $\left\{d X^{i}\right\}$ with $i=1, \ldots, D$, and expand (4.11) as

$$
\omega_{L}^{a}=\omega_{L}{ }_{i}^{a} d X^{i}, \quad \omega_{R}{ }^{a}=\omega_{R}{ }^{a}{ }_{i} d X^{i} .
$$

The dual vector fields $\xi_{L \mathrm{a}}$ and $\xi_{R \mathrm{a}}$ are defined via $\iota_{\xi_{\mathrm{a}}} \omega^{\mathrm{b}}=\delta_{\mathrm{a}}^{\mathrm{b}}$ for the left- and right-invariant sector, leading to the expressions

$$
\xi_{L \mathrm{a}}=\left(\omega_{L}^{-1}\right)^{i}{ }_{\mathrm{a}} \partial_{i}, \quad \xi_{R \mathrm{a}}=\left(\omega_{R}^{-1}\right)^{i}{ }_{\mathrm{a}} \partial_{i}
$$

They are the left- and right-invariant vector fields. For later reference, we furthermore define

$$
\iota_{\xi_{L \mathrm{a}}} \omega_{R}^{\mathrm{b}}=\omega_{R}{ }_{i}^{\mathrm{b}}\left(\omega_{L}^{-1}\right)^{i}{ }_{\mathrm{a}}=R_{\mathrm{a}}^{\mathrm{b}}, \quad \iota_{\xi_{R \mathrm{a}}} \omega_{L}^{\mathrm{b}}=\omega_{L}{ }_{i}^{\mathrm{b}}\left(\omega_{R}^{-1}\right)^{i}{ }_{\mathrm{a}}=\left(R^{-1}\right)^{\mathrm{b}}{ }_{\mathrm{a}} .
$$


The vector fields satisfy the following algebra

$$
\begin{array}{ll}
{\left[\xi_{L \mathrm{a}}, \xi_{L \mathrm{~b}}\right]=+i f_{\mathrm{ab}}{ }^{\mathrm{c}} \xi_{L \mathrm{c}},} & {\left[\xi_{L \mathrm{a}}, \xi_{R \mathrm{~b}}\right]=0,} \\
{\left[\xi_{R \mathrm{a}}, \xi_{R \mathrm{~b}}\right]=-i f_{\mathrm{ab}}{ }^{\mathrm{c}} \xi_{R \mathrm{c}},} &
\end{array}
$$

and they are Killing vectors for the metric $G$ shown in (4.12), that is

$$
\mathcal{L}_{\xi_{L \mathrm{a}}} G=0, \quad \mathcal{L}_{\xi_{R \mathrm{a}}} G=0 .
$$

This means, that the isometry group for the Lie group $G$ is $G_{L} \times G_{R}$.

\section{Gauging conditions I}

We now want to construct vector fields $k_{\alpha}$ and $v_{\alpha}$ which satisfy the gauging conditions summarized in equations (2.45) and (2.46). We make the following choice

$$
k_{\mathrm{a}}=-i\left(\xi_{L \mathrm{a}}-\xi_{R \mathrm{a}}\right), \quad v_{\mathrm{a}}=+i\left(\xi_{L \mathrm{a}}+\xi_{R \mathrm{a}}\right),
$$

for which the dual one-forms $\mathrm{k}_{\mathrm{a}}=k_{\mathrm{a}}^{i} G_{i j} d X^{j}$ and $\mathrm{v}_{\mathrm{a}}=v_{\mathrm{a}}^{i} G_{i j} d X^{j}$ are given by

$$
\mathrm{k}_{\mathrm{a}}=+i k \delta_{\mathrm{ab}}\left(\omega_{L}^{\mathrm{b}}-\omega_{R}^{\mathrm{b}}\right), \quad \mathrm{v}_{\mathrm{a}}=-i k \delta_{\mathrm{ab}}\left(\omega_{L}^{\mathrm{b}}+\omega_{R}^{\mathrm{b}}\right),
$$

where $k$ denotes again the level. Let us now discuss the implications for the gauging conditions:

- Using the properties listed above, we can show that (4.19) and (4.20) satisfy the conditions shown in (2.46).

- Furthermore, since the left- and right-invariant vectors $\xi_{L a}$ and $\xi_{R a}$ are Killing vectors, also $k_{\mathrm{a}}$ and $v_{\mathrm{a}}$ are Killing. Moreover, also the second row in (2.45) is satisfied, which can be seen by we computing

$$
d \mathrm{k}_{\mathrm{a}}-\iota_{v_{\mathrm{a}}} H=0, \quad d \mathrm{v}_{\mathrm{a}}-\iota_{k_{\mathrm{a}}} H=0 .
$$

- Finally, we have to demand that the matrix $\mathcal{G}$ defined in (2.33) is invertible. Let us therefore compute

$$
\mathcal{G}_{\mathrm{ab}}=k_{\mathrm{a}}^{i} G_{i j} k_{\mathrm{b}}^{j}=k\left[\mathbb{1}-R^{T}\right]_{\mathrm{a}}{ }^{\mathrm{c}} \delta_{\mathrm{cd}}[\mathbb{1}-R]_{\mathrm{b}}^{\mathrm{d}},
$$

where the matrix $R$ had been defined in (4.16). However, $\mathcal{G}_{\mathrm{ab}}$ with $\mathrm{a}, \mathrm{b}=$ $1, \ldots, D$ always has an eigenvector with vanishing eigenvalue 2 Hence, the condition (2.34) for invertibility of $\mathcal{G}$ is not satisfied and it is therefore not possible to gauge all isometries with Killing vectors $k_{\mathrm{a}}$ for $\mathrm{a}=1, \ldots, D$.

\footnotetext{
${ }^{2}$ Note that $R_{\mathrm{b}}^{\mathrm{a}}=\frac{1}{2} \delta^{\mathrm{ac}} \operatorname{Tr}\left(t_{\mathrm{c}} g t_{\mathrm{b}} g^{-1}\right)$, where $g \in G$. Writing then $g=\exp \left(i \phi^{\mathrm{a}} t_{\mathrm{a}}\right)$ and using the Baker-Campell-Hausdorff formula, we see that $R^{\mathrm{a}}{ }_{\mathrm{b}} \phi^{\mathrm{b}}=\phi^{\mathrm{a}}$. Hence, $(\mathbb{1}-R)^{\mathrm{a}} \mathrm{b}$ has $\phi^{\mathrm{a}}$ as an eigenvector with vanishing eigenvalue.
} 


\section{Gauging conditions II}

However, one possibility to obtain a matrix $\mathcal{G}$ which satisfies (2.34) is to not gauge all $k_{\mathrm{a}}$ for $\mathrm{a}=1, \ldots, D$, but to choose a subset. For instance, let us consider

$$
\begin{array}{ll}
k_{\alpha}=-i\left(\xi_{L \alpha}-\xi_{R \alpha}\right), & \xi_{L \alpha} \in \operatorname{Cartan}\left(\mathfrak{g}_{L}\right), \\
v_{\alpha}=+i\left(\xi_{L \alpha}+\xi_{R \alpha}\right), & \xi_{R \alpha} \in \operatorname{Cartan}\left(\mathfrak{g}_{R}\right),
\end{array}
$$

where the left- and right-invariant vector fields are elements of the Cartan subalgebra $\mathfrak{g}_{L}$ and $\mathfrak{g}_{R}$ and where $\alpha=1, \ldots, N$ with $N<D$. Since $\left[\mathfrak{g}_{L}, \mathfrak{g}_{R}\right]=0$, this implies that all $k_{\alpha}$ and $v_{\alpha}$ commute among each other

$$
\left[k_{\alpha}, k_{\beta}\right]_{\mathrm{L}}=0, \quad\left[k_{\alpha}, v_{\beta}\right]_{\mathrm{L}}=0, \quad\left[v_{\alpha}, v_{\beta}\right]_{\mathrm{L}}=0 \text {. }
$$

Furthermore, the matrix $\mathcal{G}_{\alpha \beta}$ defined in $(2.33)$ is in general invertible, except at singular points. This is precisely the setting we have encountered in section 4.1 for the $S U(2)$ case.

\section{Discussion}

In this subsection we have seen, that the example of the $S U(2)$ WZW model can be generalized to arbitrary Lie groups. For the choice of Killing vectors (4.20) the gauging conditions (2.45) and (2.46) can be satisfied, however, the matrix $\mathcal{G}_{\mathrm{ab}}$ is not invertible when gauging all $k_{\mathrm{a}}$-isometries.

On the other hand, when choosing only a subset of isometries, the matrix $\mathcal{G}_{\alpha \beta}$ is invertible except at special points. A convenient subset of isometries corresponds to the Cartan algebra, shown in (4.23). It would be interesting to further investigate this point and to construct explicit models, however, this is beyond the scope of this paper.

\section{Summary and conclusions}

In this paper, we have discussed how through a gauging procedure the number of target-space dimensions of a non-linear sigma-model can be reduced. In particular, if the parent theory exhibits target-space isometries satisfying the conditions (2.45) and (2.46), it is possible to gauge a corresponding world-sheet symmetry and integrate-out the gauge field. As we have shown, the resulting theory corresponds to a background which is reduced by one dimension. We have then generalized this procedure to gauging $N$ symmetries, resulting in reductions by $N$ dimensions.

In section 3 we have considered a particular class of geometries relevant for double field theory. In the context of T-duality, these have appeared in [6] and have 
also been used in [52]. They are characterized by the presence of an even number of abelian isometries, and can be viewed as torus fibrations over a base manifold. We have shown that these backgrounds satisfy the gauging constraints summarized in equations (2.45) and (2.46), and we have performed the corresponding reduction. Different choices for which isometries are used for the reduction lead to different child theories, which are T-dual to each other.

For the three-dimensional situation we have furthermore illustrated, that if the parent background is conformal the child theories are conformal as well. As shown in equation (3.26), the only difference occurs for the dilaton, where the corresponding $\beta$-functional of the parent theory is non-vanishing at tree-level due to not being a critical string theory. The reduced child theories on the other hand are indeed string theories with vanishing $\beta$-functionals.

In section 4 we have discussed the $S U(2)$ WZW model as an example. This model is conformal and corresponds to the three-sphere with $H$-flux, and the isometry group contains two abelian isometries. Applying the gauging procedure for one of these two isometries, leads to a conformal model in two dimensions. As it turns out, it is not possible to choose any linear combination of these isometries, but only two choices satisfy the constraints (2.45) and (2.46). These lead to the cigar and trumpet solution in two dimensions, which are again conformal models and T-dual to each other. We have furthermore commented on the generalization to WZW models on arbitrary Lie groups.

Our studies in this paper provide a starting point for the development of a world-sheet description of double field theory. The novel feature of our formalism is that the reduction from the parent theory to the child theories is realized through a gauging procedure, instead of imposing constraints explicitly. This gauging procedure should correspond to imposing the strong constraint in double field theory, which also eliminates half of the coordinates. The next task in this program is to relate the $\beta$-functionals of the world-sheet theory to the equations of motion of double field theory, which we plan to address in future work. Other open questions are to find further explicit examples where the proposed gauging reduction is realized. In particular, it would be interesting to study examples with non-abelian isometry groups. We hope to come back to these points in the future.

\section{Acknowledgements}

We thank Christoph Mayrhofer and Cornelius Schmidt-Colinet for helpful discussions. EP would like to thank the Physics Department at Seoul National University and the Erwin Schrödinger International Institute in Vienna for hospitality. This work was partially supported by the ERC Advanced Grant "Strings and Gravity" (Grant.No. 32004) and by the DFG cluster of excellence "Origin and Structure of the Universe". 


\section{A Requirement of vanishing field strength}

In this appendix we give some details on the computation leading to the constraints (2.14) and (2.36). However, since the abelian case is included in the non-abelian situation, we focus on the latter.

When gauging a sigma-model with non-abelian isometries, we impose the constraint (2.31) in order to not increase the number of degrees of freedom of the theory. As the matrix $\mathcal{M}_{\alpha \beta}$ defined in (2.33) is assumed to be invertible, we can write (2.31) also as

$$
0=\mathcal{M}_{\alpha \beta} F^{\beta}
$$

To compute $F^{\alpha}$, we need to know how the exterior derivative acts on $\star d X^{i}$ on the world-sheet. This is determined by the equation of motion of the gauged action (2.7) for $X^{i}$ which read

$$
\begin{aligned}
0= & -G_{i j}\left[\Gamma_{p q}^{j} d X^{p} \wedge \star d X^{q}+d \star d X^{j}\right] \\
& +\left(\iota_{i} d \mathrm{k}_{\alpha}\right) \wedge \star A^{\alpha}-\mathrm{k}_{\alpha i} d \star A^{\alpha}+\frac{1}{2} \partial_{i} \mathcal{G}_{\alpha \beta} A^{\alpha} \wedge \star A^{\beta} \\
& +i \iota_{i} H+i\left(\iota_{i} d \mathrm{v}_{\alpha}\right) \wedge A^{\alpha}-\frac{i}{2}\left(\iota_{i} \iota_{k_{\alpha}} \iota_{k_{\beta}} H\right) A^{\alpha} \wedge A^{\beta} \\
& +\frac{\alpha^{\prime}}{2} R \partial_{i} \Phi \star 1
\end{aligned}
$$

where the Christoffel symbols $\Gamma_{i j}^{k}$ are computed using the target-space metric $G_{i j}$. Furthermore, it is useful to note that from (2.30) we can derive the following relation

$$
0=3 \iota_{k_{[\underline{[}}} f_{\underline{\beta \gamma]}}^{\epsilon} \mathrm{v}_{\epsilon}-\iota_{k_{\alpha}} \iota_{k_{\beta}} \iota_{k_{\gamma}} H
$$

which implies

$$
\mathcal{L}_{k_{\alpha}} \mathcal{G}_{\beta \gamma}=f_{\alpha \beta}{ }^{\epsilon} \mathcal{G}_{\epsilon \gamma}+f_{\alpha \gamma}{ }^{\epsilon} \mathcal{G}_{\epsilon \beta}, \quad \mathcal{L}_{k_{\alpha}} \mathcal{D}_{\beta \gamma}=f_{\alpha \beta}{ }^{\epsilon} \mathcal{D}_{\epsilon \gamma}-f_{\alpha \gamma}{ }^{\epsilon} \mathcal{D}_{\epsilon \beta}
$$

For (A.1) we then compute

$$
\begin{aligned}
0= & \mathcal{M}_{\alpha \beta} F^{\beta} \\
= & \frac{\alpha^{\prime}}{2} R\left[v_{\alpha}^{m} \partial_{m} \Phi\right] \star 1 \\
& +\left[A_{\alpha i j} D X^{i} \wedge \star D X^{j}-2 B_{\alpha i j} D X^{i} \wedge \star(k \omega)^{j}-A_{\alpha i j}(k \omega)^{i} \wedge \star(k \omega)^{j}\right] \\
& -i\left[B_{\alpha i j} D X^{i} \wedge D X^{j}-2 A_{\alpha i j} D X^{i} \wedge(k \omega)^{j}-B_{\alpha i j}(k \omega)^{i} \wedge(k \omega)^{j}\right],
\end{aligned}
$$


where we have defined

$$
\begin{aligned}
D X^{i} & =d X^{i}-k_{\mu}^{i} l^{\mu}, & l^{\mu} & =\mathcal{M}^{\mu \nu}\left(\mathrm{k}+\mathcal{D} \mathcal{G}^{-1} \mathrm{v}\right)_{\nu}, \\
(k \omega)^{i} & =k_{\mu}^{i} \omega^{\mu}, & \omega^{\mu} & =\mathcal{M}^{\mu \nu}\left(\mathrm{v}+\mathcal{D} \mathcal{G}^{-1} \mathrm{k}\right)_{\nu},
\end{aligned}
$$

as well as

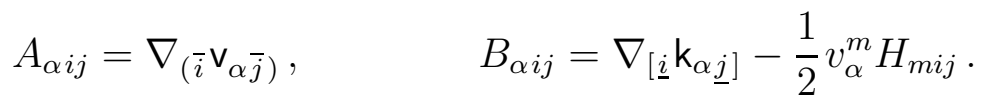

Equation (A.5) can be split into a real and imaginary part at zeroth order in $\alpha^{\prime}$, and in a part linear in $\alpha^{\prime}$. Assuming $\alpha^{\prime}$ to be an expansion parameter, we infer from the latter part that $v_{\alpha}^{m} \partial_{m} \Phi=0$. Concerning the real and imaginary part, we have not been able to show that $F^{\alpha}=0$ implies that $A_{\alpha i j}=0$ and $B_{\alpha i j}=0$. This is related to the fact that when written as matrix equations, $A_{\alpha}$ and $B_{\alpha}$ have $k_{\mu}^{i}$ as eigenvectors with vanishing eigenvalue. Furthermore, (A.5) is invariant under the following transformation

$$
\begin{aligned}
& A_{\alpha i j} \rightarrow A_{\alpha i j}+a_{\alpha}{ }^{\beta}\left(\bar{i} \mathrm{k}_{\beta \bar{j})}+b_{\alpha}{ }^{\beta}\left(\mathrm{v}_{\beta \bar{j}}\right)\right. \\
& B_{\alpha i j} \rightarrow B_{\alpha i j}+b_{\alpha}{ }^{\beta}\left[\underline{i} \mathrm{k}_{\beta} \underline{j}\right] \\
& \left.+a_{\alpha}{ }^{\beta} \mathrm{v}_{\beta} \underline{j}\right]
\end{aligned}
$$

where $a_{\alpha}{ }^{\beta}{ }_{i}$ and $b_{\alpha}{ }^{\beta}{ }_{i}$ are arbitrary functions. However, it is of course true that setting $A_{\alpha i j}$ and $B_{\alpha i j}$ to zero (and imposing $v_{\alpha}^{m} \partial_{m} \Phi=0$ ) solves $F^{\alpha}=0$. This is what we assume for this paper, namely that for all $\alpha$

$$
A_{\alpha i j}=0, \quad B_{\alpha i j}=0, \quad v_{\alpha}^{m} \partial_{m} \Phi=0,
$$

which corresponds to (2.14) and (2.36) in the main text. 


\section{References}

[1] E. Witten, "String theory dynamics in various dimensions," Nucl. Phys. B443 (1995) 85-126, hep-th/9503124.

[2] A. Giveon, M. Porrati, and E. Rabinovici, "Target space duality in string theory," Phys. Rept. 244 (1994) 77-202, hep-th/9401139.

[3] T. H. Buscher, "Quantum Corrections and Extended Supersymmetry in New $\sigma$ Models," Phys. Lett. B159 (1985) 127.

[4] T. H. Buscher, "A Symmetry of the String Background Field Equations," Phys. Lett. B194 (1987) 59.

[5] T. H. Buscher, "Path Integral Derivation of Quantum Duality in Nonlinear Sigma Models," Phys. Lett. B201 (1988) 466.

[6] M. Rocek and E. P. Verlinde, "Duality, quotients, and currents," Nucl. Phys. B373 (1992) 630-646, hep-th/9110053.

[7] A. Giveon, E. Rabinovici, and G. Veneziano, "Duality in String Background Space," Nucl. Phys. B322 (1989) 167.

[8] A. Giveon and M. Rocek, "Generalized duality in curved string backgrounds," Nucl. Phys. B380 (1992) 128-146, hep-th/9112070.

[9] A. Giveon and E. Kiritsis, "Axial vector duality as a gauge symmetry and topology change in string theory," Nucl. Phys. B411 (1994) 487-508, hep-th/9303016.

[10] E. Alvarez, L. Alvarez-Gaume, J. L. F. Barbon, and Y. Lozano, "Some global aspects of duality in string theory," Nucl. Phys. B415 (1994) 71-100, hep-th/9309039.

[11] E. Alvarez, L. Alvarez-Gaume, and Y. Lozano, "A Canonical approach to duality transformations," Phys. Lett. B336 (1994) 183-189, hep-th/9406206.

[12] I. Bakas and K. Sfetsos, "T duality and world sheet supersymmetry," Phys. Lett. B349 (1995) 448-457, hep-th/9502065.

[13] E. Plauschinn, "T-duality revisited," JHEP 01 (2014) 131, 1310.4194.

[14] E. Plauschinn, "On T-duality transformations for the three-sphere," Nucl. Phys. B893 (2015) 257-286, 1408.1715. 
[15] A. Chatzistavrakidis, A. Deser, and L. Jonke, "T-duality without isometry via extended gauge symmetries of 2D sigma models," 1509.01829.

[16] K. Dasgupta, G. Rajesh, and S. Sethi, "M theory, orientifolds and G - flux," JHEP 08 (1999) 023, hep-th/9908088.

[17] S. Kachru, M. B. Schulz, P. K. Tripathy, and S. P. Trivedi, "New supersymmetric string compactifications," JHEP 03 (2003) 061, hep-th/0211182.

[18] S. Hellerman, J. McGreevy, and B. Williams, "Geometric constructions of nongeometric string theories," JHEP 01 (2004) 024, hep-th/0208174.

[19] A. Dabholkar and C. Hull, "Duality twists, orbifolds, and fluxes," JHEP 09 (2003) 054, hep-th/0210209.

[20] C. M. Hull, "A Geometry for non-geometric string backgrounds," JHEP 10 (2005) 065, hep-th/0406102.

[21] J. Shelton, W. Taylor, and B. Wecht, "Nongeometric flux compactifications," JHEP 10 (2005) 085, hep-th/0508133.

[22] V. Mathai and J. M. Rosenberg, "T duality for torus bundles with H fluxes via noncommutative topology," Commun. Math. Phys. 253 (2004) 705-721, hep-th/0401168.

[23] V. Mathai and J. M. Rosenberg, "On Mysteriously missing T-duals, H-flux and the T-duality group," in Differential geometry and physics. Proceedings, 23rd International Conference, Tianjin, China, August 20-26, 2005, pp. 350-358. 2004. hep-th/0409073.

[24] P. Bouwknegt, K. Hannabuss, and V. Mathai, "Nonassociative tori and applications to T-duality," Commun. Math. Phys. 264 (2006) 41-69, hep-th/0412092.

[25] P. Bouwknegt, K. Hannabuss, and V. Mathai, "T-duality for principal torus bundles and dimensionally reduced Gysin sequences," Adv. Theor. Math. Phys. 9 (2005), no. 5, 749-773, hep-th/0412268.

[26] I. Ellwood and A. Hashimoto, "Effective descriptions of branes on non-geometric tori," JHEP 12 (2006) 025, hep-th/0607135.

[27] P. Grange and S. Schafer-Nameki, "T-duality with H-flux: Non-commutativity, T-folds and G x G structure," Nucl. Phys. B770 (2007) 123-144, hep-th/0609084. 
[28] R. Blumenhagen and E. Plauschinn, "Nonassociative Gravity in String Theory?," J. Phys. A44 (2011) 015401, 1010.1263.

[29] D. Lüst, "T-duality and closed string non-commutative (doubled) geometry," JHEP 12 (2010) 084, 1010.1361.

[30] R. Blumenhagen, A. Deser, D. Lüst, E. Plauschinn, and F. Rennecke, "Non-geometric Fluxes, Asymmetric Strings and Nonassociative Geometry," J. Phys. A44 (2011) 385401, 1106.0316.

[31] R. Blumenhagen, "Nonassociativity in String Theory," in Strings, gauge fields, and the geometry behind: The legacy of Maximilian Kreuzer,

A. Rebhan, L. Katzarkov, J. Knapp, R. Rashkov, and E. Scheidegger, eds., pp. 213-224. World Scientific, 2011. 1112.4611.

[32] D. Lüst, "Twisted Poisson Structures and Non-commutative/non-associative Closed String Geometry," PoS CORFU2011 (2011) 086, 1205.0100.

[33] E. Plauschinn, "Non-geometric fluxes and non-associative geometry," PoS CORFU2011 (2011) 061, 1203.6203.

[34] C. Condeescu, I. Florakis, and D. Lüst, "Asymmetric Orbifolds, Non-Geometric Fluxes and Non-Commutativity in Closed String Theory," JHEP 04 (2012) 121, 1202.6366.

[35] D. Mylonas, P. Schupp, and R. J. Szabo, "Membrane Sigma-Models and Quantization of Non-Geometric Flux Backgrounds," JHEP 09 (2012) 012, 1207.0926 .

[36] A. Chatzistavrakidis and L. Jonke, "Matrix theory origins of non-geometric fluxes," JHEP 02 (2013) 040, 1207.6412.

[37] D. Andriot, M. Larfors, D. Lüst, and P. Patalong, "(Non-)commutative closed string on T-dual toroidal backgrounds," JHEP 06 (2013) 021, 1211.6437 .

[38] I. Bakas and D. Lüst, "3-Cocycles, Non-Associative Star-Products and the Magnetic Paradigm of R-Flux String Vacua," JHEP 01 (2014) 171, 1309.3172.

[39] A. Deser, "Lie algebroids, non-associative structures and non-geometric fluxes," Fortsch. Phys. 61 (2013) 1056-1153, 1309.5792. 
[40] D. Mylonas, P. Schupp, and R. J. Szabo, "Non-Geometric Fluxes, Quasi-Hopf Twist Deformations and Nonassociative Quantum Mechanics," J. Math. Phys. 55 (2014) 122301, 1312.1621.

[41] C. D. A. Blair, "Non-commutativity and non-associativity of the doubled string in non-geometric backgrounds," JHEP 06 (2015) 091, 1405.2283.

[42] D. Mylonas, P. Schupp, and R. J. Szabo, "Nonassociative geometry and twist deformations in non-geometric string theory," PoS ICMP2013 (2013) $007,1402.7306$.

[43] P. Aschieri and R. J. Szabo, "Triproducts, nonassociative star products and geometry of R-flux string compactifications," J. Phys. Conf. Ser. 634 (2015), no. 1, 012004, 1504.03915.

[44] H. Kawai, D. C. Lewellen, and S. H. H. Tye, "Construction of Fermionic String Models in Four-Dimensions," Nucl. Phys. B288 (1987) 1.

[45] W. Lerche, D. Lüst, and A. N. Schellekens, "Chiral Four-Dimensional Heterotic Strings from Selfdual Lattices," Nucl. Phys. B287 (1987) 477.

[46] I. Antoniadis, C. P. Bachas, and C. Kounnas, "Four-Dimensional Superstrings," Nucl. Phys. B289 (1987) 87.

[47] K. S. Narain, M. H. Sarmadi, and C. Vafa, "Asymmetric Orbifolds," Nucl. Phys. B288 (1987) 551.

[48] A. Flournoy and B. Williams, "Nongeometry, duality twists, and the worldsheet," JHEP 01 (2006) 166, hep-th/0511126.

[49] N. Halmagyi, "Non-geometric String Backgrounds and Worldsheet Algebras," JHEP 07 (2008) 137, 0805.4571.

[50] N. Halmagyi, "Non-geometric Backgrounds and the First Order String Sigma Model," 0906.2891.

[51] F. Rennecke, "O(d,d)-Duality in String Theory," JHEP 10 (2014) 69, 1404.0912 .

[52] I. Bakas and D. Lüst, "T-duality, Quotients and Currents for Non-Geometric Closed Strings," Fortsch. Phys. 63 (2015) 543-570, 1505.04004.

[53] A. Chatzistavrakidis, L. Jonke, and O. Lechtenfeld, "Sigma models for genuinely non-geometric backgrounds," JHEP 11 (2015) 182, 1505.05457. 
[54] D. Andriot, M. Larfors, D. Lüst, and P. Patalong, "A ten-dimensional action for non-geometric fluxes," JHEP 09 (2011) 134, 1106.4015.

[55] D. Andriot, O. Hohm, M. Larfors, D. Lüst, and P. Patalong, "A geometric action for non-geometric fluxes," Phys. Rev. Lett. 108 (2012) 261602, 1202.3060 .

[56] D. Andriot, O. Hohm, M. Larfors, D. Lüst, and P. Patalong, "Non-Geometric Fluxes in Supergravity and Double Field Theory," Fortsch. Phys. 60 (2012) 1150-1186, 1204.1979.

[57] R. Blumenhagen, A. Deser, E. Plauschinn, and F. Rennecke, "A bi-invariant Einstein-Hilbert action for the non-geometric string," Phys. Lett. B720 (2013) 215-218, 1210.1591.

[58] R. Blumenhagen, A. Deser, E. Plauschinn, and F. Rennecke, "Non-geometric strings, symplectic gravity and differential geometry of Lie algebroids," JHEP 02 (2013) 122, 1211.0030.

[59] R. Blumenhagen, A. Deser, E. Plauschinn, F. Rennecke, and C. Schmid, "The Intriguing Structure of Non-geometric Frames in String Theory," Fortsch. Phys. 61 (2013) 893-925, 1304.2784.

[60] D. Andriot and A. Betz, " $\beta$-supergravity: a ten-dimensional theory with non-geometric fluxes, and its geometric framework," JHEP 12 (2013) 083, 1306.4381 .

[61] D. Andriot and A. Betz, "NS-branes, source corrected Bianchi identities, and more on backgrounds with non-geometric fluxes," JHEP 07 (2014) 059, 1402.5972 .

[62] A. A. Tseytlin, "Duality Symmetric Formulation of String World Sheet Dynamics," Phys. Lett. B242 (1990) 163-174.

[63] A. A. Tseytlin, "Duality symmetric closed string theory and interacting chiral scalars," Nucl. Phys. B350 (1991) 395-440.

[64] M. J. Duff, "Duality Rotations in String Theory," Nucl. Phys. B335 (1990) 610 .

[65] G. Dall'Agata and N. Prezas, "Worldsheet theories for non-geometric string backgrounds," JHEP 08 (2008) 088, 0806.2003.

[66] O. Hohm, W. Siegel, and B. Zwiebach, "Doubled $\alpha^{\prime}$-geometry," JHEP 02 (2014) 065, 1306.2970. 
[67] S. Groot Nibbelink and P. Patalong, "A Lorentz invariant doubled world-sheet theory," Phys. Rev. D87 (2013), no. 4, 041902, 1207.6110.

[68] S. Groot Nibbelink, F. Kurz, and P. Patalong, "Renormalization of a Lorentz invariant doubled worldsheet theory," JHEP 10 (2014) 114, 1308.4418.

[69] A. Dabholkar and C. Hull, "Generalised T-duality and non-geometric backgrounds," JHEP 05 (2006) 009, hep-th/0512005.

[70] C. M. Hull, "Doubled Geometry and T-Folds," JHEP 07 (2007) 080, hep-th/0605149.

[71] W. Siegel, "Two vierbein formalism for string inspired axionic gravity," Phys. Rev. D47 (1993) 5453-5459, hep-th/9302036.

[72] W. Siegel, "Superspace duality in low-energy superstrings," Phys. Rev. D48 (1993) 2826-2837, hep-th/9305073.

[73] C. Hull and B. Zwiebach, "Double Field Theory," JHEP 09 (2009) 099, 0904.4664 .

[74] O. Hohm, C. Hull, and B. Zwiebach, "Background independent action for double field theory," JHEP 07 (2010) 016, 1003.5027.

[75] O. Hohm, C. Hull, and B. Zwiebach, "Generalized metric formulation of double field theory," JHEP 08 (2010) 008, 1006.4823.

[76] G. Aldazabal, D. Marques, and C. Nunez, "Double Field Theory: A Pedagogical Review," Class. Quant. Grav. 30 (2013) 163001, 1305.1907.

[77] O. Hohm, D. Lüst, and B. Zwiebach, "The Spacetime of Double Field Theory: Review, Remarks, and Outlook," Fortsch. Phys. 61 (2013) 926-966, 1309.2977.

[78] K. Hori, "D-branes, T duality, and index theory," Adv. Theor. Math. Phys. 3 (1999) 281-342, hep-th/9902102.

[79] P. Bouwknegt, J. Evslin, and V. Mathai, "T duality: Topology change from H flux," Commun. Math. Phys. 249 (2004) 383-415, hep-th/0306062.

[80] N. Hitchin, "Generalized Calabi-Yau manifolds," Quart. J. Math. 54 (2003) 281-308, math/0209099.

[81] M. Gualtieri, Generalized complex geometry. PhD thesis, Oxford U., 2003. math/0401221. 
[82] M. Grana, R. Minasian, M. Petrini, and D. Waldram, "T-duality, Generalized Geometry and Non-Geometric Backgrounds," JHEP 04 (2009) $075,0807.4527$.

[83] C. M. Hull and B. J. Spence, "The Gauged Nonlinear $\sigma$ Model With Wess-Zumino Term," Phys. Lett. B232 (1989) 204.

[84] C. M. Hull and B. J. Spence, "The Geometry of the gauged sigma model with Wess-Zumino term," Nucl. Phys. B353 (1991) 379-426.

[85] R. Blumenhagen, F. Hassler, and D. Lüst, "Double Field Theory on Group Manifolds," JHEP 02 (2015) 001, 1410.6374.

[86] P. d. Bosque, F. Hassler, and D. Lüst, "Flux Formulation of DFT on Group Manifolds and Generalized Scherk-Schwarz Compactifications," JHEP 02 (2016) 039, 1509.04176. 